\title{
Stress Index Can Be Accurately and Reliably Assessed by Visually Inspecting Ventilator Waveforms
}

\author{
Xiu-Mei Sun MSc, Guang-Qiang Chen MD, Kai Chen MD, Yu-Mei Wang MD, Xuan He MSc, \\ Hua-Wei Huang MD, Xu-Ying Luo MD, Chun-Mei Wang MD, Zhong-Hua Shi MD, \\ Ming $\mathrm{Xu}$ MD, Lu Chen MD, Eddy Fan MD PhD, and Jian-Xin Zhou MD
}

\begin{abstract}
BACKGROUND: Stress index provides a noninvasive approach to detect injurious ventilation patterns and to personalize ventilator settings. Obtaining the stress index (SI), however, requires quantitatively analyzing the shape of pressure-time curve with dedicated instruments or a specific ventilator, which may encumber its clinical implementation. We hypothesized that the SI could be qualitatively determined through a visual inspection of ventilator waveforms. METHODS: Thirtysix adult subjects undergoing volume controlled ventilation without spontaneous breathing were enrolled. For each subject, 2 trained clinicians visually inspected the pressure-time curve directly from the ventilator screen. They then qualitatively categorized the shape of pressure-time curve as linear, a downward concavity, or an upward concavity at the bedside. We simultaneously recorded airway pressure and flow signals using a dedicated instrument. A quantitative off-line analysis was performed to calculate the SI using specific research software. This quantitative analysis of the SI served as the reference method for classifying the shape of the pressure-time curve (ie, linear, a downward concavity, or an upward concavity). We compared the SI categorized by visual inspection with that by the reference. RESULTS: We obtained 200 SI assessments of pressure-time curves, among which $125(63 \%)$ were linear, $55(27 \%)$ were a downward concavity, and $20(10 \%)$ were an upward concavity as determined by the reference method. The overall accuracy of visual inspection and weighted kappa statistic $(95 \%$ CI) was $93 \%(88-96 \%)$ and $0.88(0.82-0.94)$, respectively. The sensitivity and specificity to distinguish a downward concavity from a linear shape were $91 \%$ and $98 \%$, respectively. The respective sensitivity and specificity to distinguish an upward concavity from a linear shape were $95 \%$ and $95 \%$. CONCLUSIONS: Visual inspection of the pressure-time curve on the ventilator screen is a simple and reliable approach to assess SI at the bedside. This simplification may facilitate the implementation of SI in clinical practice to personalize mechanical ventilation. (ClinicalTrials.gov registration NCT03096106.) Key words: mechanical ventilation; respiratory mechanics; stress index; ventilator-induced lung injury; personalized medicine. [Respir Care 2018;63(9):1094-1101. (C) 2018 Daedalus Enterprises]
\end{abstract}

\section{Introduction}

Mechanical ventilation, while providing necessary ventilatory support and gas exchange, may induce lung in-

Drs GQ Chen, K Chen, Y-M Wang, H-W Huang, X-Y Luo, C-M Wang, Z-H Shi, M Xu, and J-X Zhou, along with X-M Sun and X He, are affiliated with the Department of Critical Care Medicine, Beijing Tiantan Hospital, Capital Medical University, Beijing, China. Dr L Chen is affiliated with Keenan Research Centre, Li Ka Shing Knowledge Institute, St. Michael's Hospital, Toronto, Ontario, Canada. Drs L Chen and Fan jury. ${ }^{1}$ Alveolar overdistention and tidal recruitment are 2 main mechanisms of ventilator-induced lung injury. ${ }^{2}$ To limit the risk of overdistention and tidal recruitment, tidal volume $\left(\mathrm{V}_{\mathrm{T}}\right)$ and PEEP are adjusted. ${ }^{3,4}$ However, opti- 
mizing $\mathrm{V}_{\mathrm{T}}$ and PEEP for each individual patient is challenging, partly due to the lack of an easy-to-use method to evaluate overdistention and tidal recruitment at the bedside. $^{5}$

\section{See the Related Editorial on Page 1194}

Assessing respiratory mechanics is essential to individualize ventilator settings to minimize ventilator-induced lung injury. ${ }^{6}$ Analysis of the dynamic airway pressure-time $\left(\mathrm{P}_{\mathrm{aw}}-\mathrm{t}\right)$ curve profile is a noninvasive, real-time method for respiratory monitoring at the bedside. Since it was first introduced in $2000,{ }^{7}$ analysis of $\mathrm{P}_{\mathrm{aw}}$-t curve profiles has been used to identify tidal recruitment and overdistention in both animal experiments and clinical studies. ${ }^{7-16}$ During volume controlled ventilation, the shape of the $P_{a w}-t$ curve within the constant inspiratory flow section is related to the change of respiratory system elastance. $\mathrm{P}_{\mathrm{aw}}-\mathrm{t}$ curves that are linear, have a downward concavity, or have an upward concavity indicate that the elastance is constant, decreasing, or increasing during tidal inspiration, respectively. ${ }^{17,18}$ The stress index (SI), which is a dimensionless coefficient derived from curve fitting, can quantitatively describe the shape of the $\mathrm{P}_{\mathrm{aw}}$-t curve. However, it requires dedicated data acquisition instruments and analytic software. To our knowledge, there is only one type of ventilator that incorporates the measurement of SI (Servo-i, Maquet, Solna, Sweden) which requires a pre-installed software module. The requirements for specific hardware and software may limit the implementation and use of SI in clinical practice. Some academic centers in the United States have incorporated visual inspection of SI at the bedside. To our knowledge, however, a systematic visual method has not been reported and the accuracy of such a simplified approach has never been validated.

Because different SI values correspond to different $\mathrm{P}_{\mathrm{aw}}-\mathrm{t}$ curve shapes, we speculated that SI could be qualitatively assessed by visually inspecting the waveforms on a ventilator screen. A qualitative assessment would be sufficient to identify injurious ventilation patterns such as overdistention and tidal recruitment. Our aim was to provide a simple and reliable method for personalizing ventilator settings at the bedside. We tested the accuracy and the

Supplementary material related to this paper is available at http:// www.rcjournal.com.

Correspondence: Jian-Xin Zhou MD, Department of Critical Care Medicine, Beijing Tiantan Hospital, Capital Medical University, No 6, Tiantan Xili, Dongcheng district, 100050, Beijing, China. E-mail: zhoujx.cn@icloud.com.

DOI: $10.4187 /$ respcare.06151

\section{QUICK LOOK}

\section{Current knowledge}

Mechanical ventilation provides ventilatory and gas exchange support but may also induce lung injury. The stress index provides a noninvasive approach to detect injurious ventilation patterns and to personalize ventilator settings. However, obtaining the stress index requires quantitatively analyzing the shape of airway pressure-time curve with specific analytical instruments, which may limit its clinical implementation.

\section{What this paper contributes to our knowledge}

Our study describes a valid and reliable method for visual inspection of stress index. The results support the suggestion that this simple approach can be utilized quickly and accurately at the bedside to provide optimal, personalized ventilation.

inter-observer reliability of the visual inspection method in this study.

\section{Methods}

\section{Study Ethics and Population}

This prospective observational study was conducted in the neurosurgical ICU of the Beijing Tiantan Hospital, Capital Medical University, Beijing, China. The protocol was approved by the local institutional review board (KY 2017-016-02) and was registered at ClinicalTrials.gov (NCT03096106). Written informed consent was obtained from each subject's appropriate substitute decision maker because the subject was unconsciousness at enrollment.

Patients were screened daily at 10:00 Am for study eligibility. The same subject was allowed to test repeatedly on different days. Inclusion criteria were age $18-80 \mathrm{y}$, intubated and ventilated with volume controlled ventilation using constant flow, and deep sedation and absence of spontaneous breathing (ie, no triggering during tidal breaths and no inspiratory effort during a 5-s end-expiratory hold). Exclusion criteria were evidence of active air leak from the lung, including bronchopleural fistula, pneumothorax, pneumomediastinum, or existing chest tube; chest wall and/or abdominal injuries; and evidence suggesting reduced chest wall compliance, such as existing large pleural effusion, thoracic trauma, and or intraabdominal hypertension (ie, intra-abdominal pressure $>20 \mathrm{~mm} \mathrm{Hg}$ ). 


\section{Ventilator WAVEFORMS AND STREsS INDEX}
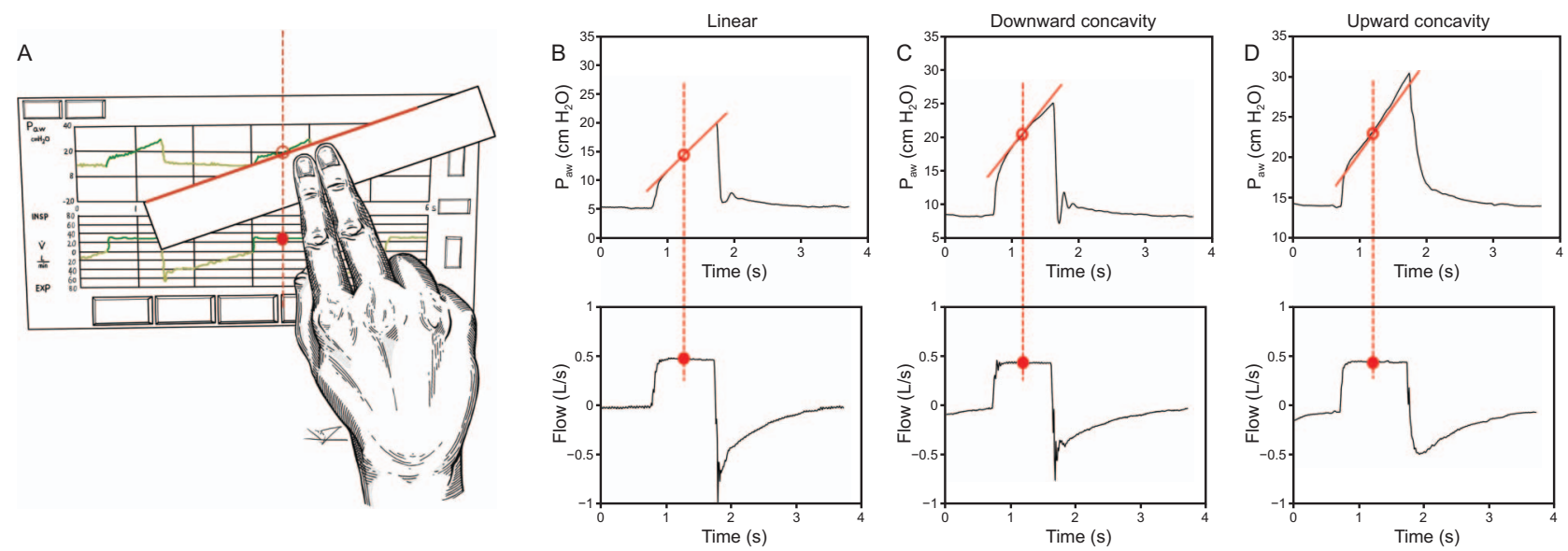

Fig. 1. Schematic of the method for visually inspecting the airway pressure-time $\left(\mathrm{P}_{\mathrm{aw}}-\mathrm{t}\right)$ waveform and stress index (SI) classification. First, the mid-point on the constant inspiratory flow (red dot) is identified; second, the corresponding point (red dashed line and red circle) on the $\mathrm{P}_{\mathrm{aw}}$ - $\mathrm{t}$ waveform is confirmed; third, a ruler is put on the ventilator screen to mark the tangent line (red solid line) passing through the middle point (A). The relationship of this tangent line and the $P_{a w}-t$ waveform is visually inspected and classified into 3 categories. The $P_{a w}-t$ waveform almost coincident with the tangent line is judged as a linear shape (B), indicating an SI value between 0.9 and 1.1. The off-line software measured $\mathrm{SI}$ to be 0.98 in this case. When the 2 sides of the $\mathrm{P}_{\mathrm{aw}}$-time waveform both deviate downward from the tangent line, this is categorized as a downward concavity (C), indicating an $\mathrm{SI}<0.9$. The off-line software measured $\mathrm{SI}$ to be 0.80 in this case. When the 2 sides of the $P_{a w}$-time waveform both deviate upward from the tangent line, this is categorized as an upward concavity (D), indicating an $\mathrm{SI}>1.1$. The off-line software measured $\mathrm{SI}$ to be 1.20 in this case.

\section{$P_{a_{w}}-t$ Curve Analysis and SI Classification With Dedicated Software}

After enrollment, a heated Fleisch pneumotachograph (Vitalograph, Lenexa, Kansas) was placed between the Y-piece of the ventilator circuit and the endotracheal tube to measure flow. A KT 100D-2 pressure transducer (KleisTEK, Bari, Italy) was connected proximally to the endotracheal tube to measure the $\mathrm{P}_{\mathrm{aw}}$. Signals were continuously displayed and saved (ICU-Lab 2.5 software package, KleisTEK) on a laptop computer for further analysis, at a sample rate of $200 \mathrm{~Hz}$.

The $\mathrm{P}_{\mathrm{aw}}$-t curve profile analysis was performed using the standard method (see the supplementary materials at http://www.rcjournal.com)..$^{7,10,13,15}$ The constant flow segment was identified as the portion of the waveform where the fluctuation was within $\pm 3 \%$ of the steady value. To eliminate the influence of on-flow and off-flow transients, the segment was further narrowed for $50 \mathrm{~ms}$ after the beginning and before the end of the constant flow portion (see the supplementary materials). If a constant segment could not be found or the length of selected segment was less than one third of the entire inspiratory phase, the data were discarded but documented. After confirming the constant flow segment, SI was automatically calculated by fitting the $\mathrm{P}_{\mathrm{aw}}$-t curve within the corresponding segment (see the supplementary materials). ${ }^{7}$ The shape of each $\mathrm{P}_{\mathrm{aw}}-\mathrm{t}$ curve was classified into 3 categories according to the SI value: a linear shape with SI $=0.9-1.1$, a downward concavity shape with SI $<0.9$, and an upward concavity shape with SI $>1.1$ (see the supplementary materials). ${ }^{18}$

\section{Visual Classification of SI and Training}

A standardized method was developed for visual SI estimation by inspecting the waveforms on the ventilator screen (Fig. 1). The midpoint on the constant inspiratory flow was identified, and then the corresponding point on the $\mathrm{P}_{\mathrm{aw}}-\mathrm{t}$ waveform was confirmed. We then put a ruler on the ventilator screen, with the straight edge passing through the point as the reference of tangent line of the $\mathrm{P}_{\mathrm{aw}}-\mathrm{t}$ waveform (Fig. 1A). The relationship of this ruler and the $\mathrm{P}_{\mathrm{aw}}$-t waveform was visually inspected and classified as a linear shape if the $\mathrm{P}_{\mathrm{aw}}-\mathrm{t}$ waveform almost coincident with the ruler, indicating an SI $=0.9-1.1$ (Fig. 1B); as a downward concavity if the 2 sides of the $\mathrm{P}_{\mathrm{aw}}-\mathrm{t}$ waveform both deviated downward from the ruler and the curve was judged as a downward concavity shape, indicating an SI $<0.9$ (Fig. 1C); or as an upward concavity if the 2 sides of the $\mathrm{P}_{\mathrm{aw}}-\mathrm{t}$ waveform both deviated upward from the ruler and the curve was judged as an upward concavity shape, indicating an SI > 1.1 (Fig. 1D).

Four critical care physicians were trained to perform the visual classification. For the pre-test training, 20 subjects' data with ventilator screen photographs and off-line SI analysis were collected. One expert conducted 2 rounds of group training. The observers were asked to discriminate the shape of the $\mathrm{P}_{\mathrm{aw}}$-t waveform as linear, a downward concavity, or an upward concavity with a training set of ventilator screen photographs. During group training, the observers reported that it was difficult to distinguish the upward concavity shape from the linear shape. A special instruction was emphasized that the upward concavity shape 
should only be confirmed when both sides of the $\mathrm{P}_{\mathrm{aw}}-\mathrm{t}$ waveform deviated from the tangent line (ruler) (Fig. 1D). The training manual is available in the supplementary materials.

\section{On-Site Visual SI Classification and Data Collection}

After enrollment, we collected the following information: ventilator brands and clinical settings, and subjects' baseline characteristics and physiological data. Three ventilator brands were used in our unit: Servo-i (Maquet), Avea (CareFusion, Mettawa, Illinois), and Puritan Bennett 840 (Covidien, Tyco Healthcare International Trading, China). The investigators were not involved in any clinical decisions. Ventilator settings were not changed during the on-site test. The data acquisition system was set up, and flow and $\mathrm{P}_{\mathrm{aw}}$ tracings were closely observed to confirm that the subject did not have any spontaneous breathing efforts. A 5-s end-expiratory occlusion was performed, and the ventilator screen displaying flow-time and $\mathrm{P}_{\mathrm{aw}}-\mathrm{t}$ waveforms was frozen to ensure that the screen contained the entire breath just before the occlusion, which was used as the sample breath for visual SI classification and subsequent off-line software analysis. The test was terminated if the subject exhibited spontaneous inspiratory effort during occlusion. In each on-site assessment, 2 observers were randomly chosen from the 4 trained physicians to independently perform visual SI classification at the bedside using our method. The trained observers were instructed to classify the sample breath as having a linear, a downward concavity, or an upward concavity shape, and the results were documented.

Flow and $\mathrm{P}_{\mathrm{aw}}$ data were collected for off-line analysis. SI was measured by an experienced investigator, who was blinded to the result of visual classification. The same sample breath used for the visual classification was used for the quantitative analysis, (ie, the breath just before the end-expiratory occlusion). Therefore, for each tested breath, on-site visual SI classifications by the 2 observers were accompanied by an off-line software fitting result. In the first 50 off-line analyses, we also measured the averaged SI value during the 2-min period before the sample breath.

\section{Sample Size}

Sample size was calculated using Power Analysis and Sample Size (PASS 11, NCSS, Kaysville, Utah). The primary end point was the agreement between the visual SI classification and the reference. Our pre-training pilot data showed that the prevalence of linear, downward concavity, and upward concavity $\mathrm{P}_{\mathrm{aw}}$-t curves using off-line software fitting was $75 \%, 15 \%$, and 10\%, respectively. Thus, 192 assessments were required to reject the null hypothesis that the kappa statistic was equal to 0.4 for a 2 -tailed test with the type- 1 error probability of 0.05 and type- 2 error probability of 0.2 . To compensate for unqualified data, 220 assessments were collected in the formal test.

\section{Statistical Analysis}

SI assessed by the off-line software fitting was used as the reference standard. Agreement between the visual SI classification and the reference standard, as well as interobserver reliability, was evaluated using a weighted kappa statistic and $95 \%$ CI. A weighted kappa statistic $>0.80$ is considered as excellent agreement. ${ }^{19}$ For the first and the second on-site observer, the result with the higher weighted kappa statistic was used for further analysis of diagnostic accuracy. The overall accuracy (95\% CI) for visual SI classification was reported. The chi-square test was used to compare the accuracy among the 3 SI classifications and among different ventilator brands. With respect to the reference, sensitivity, specificity, positive predictive value, and negative predictive value for visual SI classification to distinguish downward concavity or upward concavity, the deviation of the $\mathrm{P}_{\mathrm{aw}}$ - $\mathrm{t}$ waveform shape from a linear shape was calculated..$^{20}$ In particular, the diagnostic accuracy of each and combined upward concavity and downward concavity classification were assessed against the classification of linear shape. Statistical analyses were conducted using SPSS V.20.0 (SPSS, Chicago, Illinois). A $P<.05$ was considered statistically significant.

\section{Results}

We enrolled 36 subjects and collected 220 SI assessments (Table 1); 10 assessments were excluded due to spontaneous breathing effort after enrollment, and another 10 assessments were excluded as the length of the constant flow segment was less than one third of the inspiratory phase (see the supplementary materials). We eventually analyzed 200 qualified assessments obtained from Servo-i (41\%), Avea (31\%), and Puritan Bennett 840 ventilator (29\%). The median (interquartile range) of tidal volume $\left(\mathrm{V}_{\mathrm{T}}\right)$ per predicted body weight and PEEP were $7.2(6.5-$ 8.6) $\mathrm{mL} / \mathrm{kg}$ and $8(6-10) \mathrm{cm} \mathrm{H}_{2} \mathrm{O}$, respectively. Using off-line software analysis (reference standard), the values of SI were classified as having a linear, a downward concavity, or an upward concavity shape in $125(63 \%)$, $55(27 \%)$, and $20(10 \%)$ assessments, respectively (Table 2). In the first 50 off-line analyses, the $P_{a w}-t$ curve shape assessed by the SI value of the single sampled breath was identical to that determined with the 2-min mean value in each assessment (see the supplementary materials).

Agreement between visual SI classification by the first and the second observer and the reference standard was substantial, with weighted kappa $(95 \% \mathrm{CI})$ being 0.86 (0.80-0.92) and $0.88(0.82-0.94)$, respectively. Inter-ob- 
Table 1. Subjects' Characteristics

\begin{tabular}{|c|c|}
\hline \multicolumn{2}{|l|}{ Characteristics } \\
\hline Age, y & $40(38-58)$ \\
\hline Male & $22(61)$ \\
\hline Height, cm & $172(168-175)$ \\
\hline Weight, kg & $71(60-80)$ \\
\hline Predicted body weight, $\mathrm{kg}$ & $69(58-71)$ \\
\hline Body mass index, $\mathrm{kg} / \mathrm{m}^{2}$ & $25(20-27)$ \\
\hline \multicolumn{2}{|l|}{ Blood gas analysis at ICU admission } \\
\hline $\mathrm{pH}$ & $7.45(7.38-7.47)$ \\
\hline $\mathrm{P}_{\mathrm{aCO}_{2}}, \mathrm{~mm} \mathrm{Hg}$ & $40(33-40)$ \\
\hline $\mathrm{P}_{\mathrm{aO}_{2}}, \mathrm{~mm} \mathrm{Hg}$ & $120(94-133)$ \\
\hline $\mathrm{P}_{\mathrm{aO}_{2}} / \mathrm{F}_{\mathrm{IO}_{2}}$ & $290(224-385)$ \\
\hline APACHE II at ICU admission & $14(12-15)$ \\
\hline \multicolumn{2}{|l|}{ Diagnosis } \\
\hline Severe brain injury & $18(50)$ \\
\hline Postoperative & $10(28)$ \\
\hline $\mathrm{ARF}$ & $8(22)$ \\
\hline Assessments per subject, no. & $6(4-7)$ \\
\hline \multicolumn{2}{|c|}{$\begin{array}{l}\text { Data are presented as number }(\%) \text { or medians (interquartile ranges) when appropriate. } N=36 . \\
\text { APACHE }=\text { Acute Physiology and Chronic Health Evaluation } \\
\text { ARF }=\text { acute respiratory failure }\end{array}$} \\
\hline
\end{tabular}

server reliability was near perfect, with weighted kappa (95\% CI) 0.96 (0.92-0.99). Thus, results from the second observer were used for further analysis.

The overall accuracy $(95 \% \mathrm{CI})$ for visual SI classification was $93 \%$ (88-96\%). There was no significant difference in accuracy among reference SI classifications $(P=.20)$ or among different ventilator brands $(P=.68)$ (see the supplementary materials).

The contingency table for diagnostic accuracy analysis is shown in the supplementary materials. The sensitivity, specificity, positive predictive value, and negative predictive value of visually detecting a downward concavity shape as compared to a linear shape were $91 \%, 98 \%, 96 \%$, and $96 \%$; these respective values for visually detecting an upward concavity shape as compared to a linear shape were $95 \%, 95 \%, 76 \%$, and 99\% (Table 3). When reference classifications of a downward concavity and an upward concavity were combined as the positive result, the sensitivity and specificity for visual SI classification were $92 \%$ and $94 \%$, respectively.

\section{Discussion}

In this study, we introduced a method for estimating SI at the bedside by visually inspecting the waveforms on the ventilator screen. Our main finding is that visual SI classification shows an excellent agreement with the reference method, which used off-line software fitting. The visual inspection method can be used to simply, accurately, and reliably distinguish the $\mathrm{P}_{\mathrm{aw}}$-t curve shapes, which can help to identify potentially injurious ventilator settings in a timely manner at the bedside.

The $\mathrm{P}_{\mathrm{aw}}$-t curve profile analysis can be used to guide $\mathrm{V}_{T}$ and PEEP settings during mechanical ventilation. ${ }^{17,18} \mathrm{Ex}-$ periments in a porcine acute lung injury model showed that an $\mathrm{SI}<0.9$ indicated tidal recruitment and an $\mathrm{SI}>1.1$ indicated overdistention. ${ }^{10,11}$ Moreover, histologic and inflammatory markers of injurious ventilation were minimized when the ventilator was set to maintain SI in the range of 0.9-1.1.7-9 In subjects with ARDS, titrating PEEP to obtain SI of 0.9-1.1 attenuated alveolar overdistention, decreased lung elastance, and increased recruitment volume and oxygenation. ${ }^{13,14}$ Based on these results, we selected 0.9 and 1.1 as the thresholds for SI classification in our study.

Several conditions are required for accurate and reliable $\mathrm{P}_{\mathrm{aw}}$-t curve analysis. ${ }^{17,18} \mathrm{We}$ excluded patients with symptoms of impaired chest wall elastance, which may influence the SI interpretation. ${ }^{21}$ A standard algorithm was used for off-line software fitting at a high sample rate $(200 \mathrm{~Hz})$. A stable constant flow inspiration is critical for the SI determination. ${ }^{17,18}$ One of the disadvantages of SI is that it cannot be measured under spontaneous breathing. Thus, we excluded the presence of spontaneous effort by performing an end-expiratory occlusion before each assessment of SI. In previous reports, flow and pressure data were recorded for at least $2 \mathrm{~min}$ to obtain a mean SI value. ${ }^{7,10,15}$ However, only a single breath can be visually inspected on the ventilator screen. In a pre-specified analysis, we found identical SI classifications determined with a single sample breath and with the mean SI value of 2-min recorded breaths, which supports the ability to analyze only a single breath. Using $\mathrm{P}_{\mathrm{aw}}$-t curve to assess the change in compliance assumes that the resistive pressure remains unchanged at a constant flow during inflation. This assumption can be challenged in a few circumstances when airway resistance is varied during inflation, such as airway closure and reopening. We therefore decided to test the accuracy of SI assessment using clinical ventilator settings with various flow and PEEP levels. We did not set a low flow (eg, $<10 \mathrm{~L} / \mathrm{min}$ ) to assess SI in our study. Although the low flow may minimize the resistive pressure and might accentuate the shape for analysis, there are several practical issues and a theoretic issue that preclude us from using it. First, air leak, especially micro leaks around the endotracheal cuff and circuit connections, are extremely difficult to eliminate completely in clinical practice. Using a low flow can significantly amplify the air leak because the inspiratory time is much longer. In this case, any drop on the $\mathrm{P}_{\mathrm{aw}}$ during inflation can greatly influence the shape of $\mathrm{P}_{\mathrm{aw}}$-t curve, leading to potential misinterpretations. Second, flow sensors on ventilators are usually less accurate and less sensitive to a low flow. Third, we intentionally kept the assessment as simple as possible (ie, without the 
Table 2. Characteristics of Qualified Breaths for Off-Line Analysis (no. = 200)

\begin{tabular}{|c|c|c|c|}
\hline \multirow{2}{*}{ Characteristics } & \multicolumn{3}{|c|}{ Stress Index Categories } \\
\hline & Linear $($ no. $=125,63 \%)$ & Downward Concavity $($ no. $=55,27 \%)$ & Upward Concavity (no. $=20,10 \%$ ) \\
\hline Stress index & $0.96(0.91-1.00)$ & $0.82(0.80-0.85)$ & $1.12(1.12-1.17)$ \\
\hline \multicolumn{4}{|l|}{ Ventilators } \\
\hline Servo-i & $47(58)$ & $22(27)$ & $12(15)$ \\
\hline Avea & $37(60)$ & $21(34)$ & $4(6)$ \\
\hline PB 840 & $41(72)$ & $12(21)$ & $4(7)$ \\
\hline \multicolumn{4}{|l|}{ Ventilator settings } \\
\hline f, breaths/min & $20(16-20)$ & $20(18-22)$ & $18(18-18)$ \\
\hline $\mathrm{V}_{\mathrm{T}}, \mathrm{mL}$ & $550(500-580)$ & $365(340-450)$ & $695(635-735)$ \\
\hline $\mathrm{V}_{\mathrm{T}}, \mathrm{mL} / \mathrm{kg} \mathrm{PBW}$ & $7.2(6.5-8.6)$ & $5.9(5.1-7.1)$ & $9.3(8.5-10.1)$ \\
\hline PEEP, $\mathrm{cm} \mathrm{H}_{2} \mathrm{O}$ & $8(6-10)$ & $5(3-6)$ & $12(12-13)$ \\
\hline Inspiratory time, $\mathrm{s}$ & $0.9(0.8-1.0)$ & $0.7(0.5-1.0)$ & $1.0(1.0-1.0)$ \\
\hline Inspiratory flow, $\mathrm{L} / \mathrm{min}$ & $40(34-42)$ & $38(28-40)$ & $41(40-44)$ \\
\hline Length of selected segment, $\mathrm{s}$ & $0.5(0.5-0.7)$ & $0.3(0.3-0.6)$ & $0.5(0.5-0.6)$ \\
\hline$\%$ of entire inspiratory time & $63.5(56.3-67.9)$ & $53.1(45.9-62.3)$ & $52.9(48.5-60.2)$ \\
\hline$R$ for curve fitting & $1.00(0.99-1.00)$ & $0.99(0.99-1.00)$ & $0.99(0.99-1.00)$ \\
\hline $\begin{array}{l}\text { Data are presented as number }(\%) \text { or med } \\
\mathrm{f}=\text { breathing frequency } \\
\mathrm{V}_{\mathrm{T}}=\text { tidal volume } \\
\mathrm{PBW}=\text { predicted body weight } \\
R=\text { correlation coefficient }\end{array}$ & nterquartile range) when appropriate & & \\
\hline
\end{tabular}

Table 3. Diagnostic Accuracy Measures of Visual Stress Index Classification for Detecting Downward Concavity and/or Upward Concavity Shape of Airway Pressure-Time Curve

\begin{tabular}{lccc}
\hline \hline \multicolumn{1}{c}{ Parameters } & $\begin{array}{c}\text { Downward Concavity } \\
\text { Against Linear }\end{array}$ & $\begin{array}{c}\text { Upward Concavity } \\
\text { Against Linear }\end{array}$ & $\begin{array}{c}\text { Combined Downward Concavity and } \\
\text { Upward Concavity Against Linear }\end{array}$ \\
\hline Sensitivity, \% (95\% CI) & $91(83-99)$ & $95(85-100)$ & $92(86-98)$ \\
Specificity, \% (95\% CI) & $98(96-100)$ & $95(92-99)$ & $94(89-98)$ \\
PPV, \% (95\% CI) & $96(91-100)$ & $76(59-93)$ & $90(83-96)$ \\
NPV, \% (95\% CI) & $96(93-100)$ & $99(98-100)$ & $95(91-99)$ \\
PPV = positive predictive value & & & \\
NPV = negative predictive value & & & \\
\hline
\end{tabular}

need to change the ventilator settings for clinicians). Finally, the alveolar recruitment process may depend on the different time constant in different lung regions, and a tidal recruitment that occurs in a low-flow inflation does not necessarily mean it would occur in a regular flow. Similarly, overdistention during a regular flow inflation does not necessarily mean it would occur in a low-flow inflation.

The $\mathrm{P}_{\mathrm{aw}}-\mathrm{t}$ curve may change from a linear shape to an upward concavity or to a downward concavity depending on the amount of $\mathrm{V}_{\mathrm{T}}$ delivered. ${ }^{10} \mathrm{We}$ did not alter the clinical settings of the ventilator during the study. In addition, the delivered $\mathrm{V}_{\mathrm{T}}$ was higher in the upward concavity category than in the downward concavity and linear categories (Table 2). During mechanical ventilation, a patient's respiratory mechanics may change dynamically over time. Thus, repeated adjustments of ventilator settings are needed to minimize the risks and prevent ventilator-induced lung injury. Simple bedside monitoring that provides real-time answers is required for a method to be useful to clinicians. Our results confirmed the hypothesis that SI can be assessed by visually inspecting the waveforms on the ventilator screen. Visual SI classification yielded excellent agreement with off-line software determination, excellent inter-observer reliability, and high overall accuracy independent of SI classifications and ventilator brands. There are 3 core components in our visual SI classification method (Fig. 1). First, confirmation of the midpoint on a square flow waveform ensures to the greatest extent possible that the period with steady-state flow is selected for visual inspection. Second, placing a ruler on the ventilator screen to mark the tangent line is easy to do 


\section{Ventilator WaVeforms AND StREss IndEX}

at the bedside. Third, visually comparing the shape of the pressure waveform with the tangent line is the key step. During group training, we noticed that mistakes were more likely to occur in distinguishing the upward concavity shape from the linear shape. The observers were specifically instructed that the upward concavity shape should only be confirmed when both sides of the $\mathrm{P}_{\mathrm{aw}}$-t waveform deviate from the tangent line (Fig. 1D). Nonetheless, although acceptable sensitivity and specificity were obtained, a low positive predictive value (Table 3 ) was found in the use of visual classification to detect an upward concavity shape against a linear shape during the formal on-site test, which suggested that training should be emphasized on the identification of an upward concavity shape.

For $\mathrm{P}_{\mathrm{aw}}-\mathrm{t}$ curve profile analysis, the clinical condition of interest lies in its ability to quickly and accurately detect the shape that is consistent with "non-protective" ventilator settings. ${ }^{15}$ Using software-derived SI classification as the reference, excellent diagnostic accuracy was found for this visual inspection method in distinguishing the downward or upward concavity shape from the linear shape. A single visual classification typically took $<30 \mathrm{~s}$, which may facilitate the process of monitoring and ventilator settings titration at the bedside. Further studies are needed to investigate the clinical feasibility of the visual inspection method in the adjustment of ventilator settings.

There are several limitations to our study. First, the inspiratory flow was set at approximately $40 \mathrm{~L} / \mathrm{min}$ during our routine clinical practice. We did not test the visual inspection method at higher flows. Second, the identification of a stable flow segment is critical in $\mathrm{P}_{\mathrm{aw}}-\mathrm{t}$ curve analysis. ${ }^{17,18}$ An unqualified constant flow segment occurred in nearly 5\% (10 of 220 assessments) in our off-line fitting, but this situation was not observed during on-site visual inspection. Third, whereas a sigmoid shape of the $\mathrm{P}_{\mathrm{aw}}-\mathrm{t}$ curve has been found in animal models of ARDS,, 910 we did not find an obviously sigmoid-shaped curve during visual inspection or with off-line nonlinear fitting, possibly due to the low $\mathrm{V}_{\mathrm{T}}$ that we used. Alternatively, this difference may result from the degree of the lung inhomogeneity. We speculate that the lungs were relatively homogenous in our subjects, most of whom were surgical and neurological patients. In patients with ARDS who have more inhomogeneous lungs, it is possible that a sigmoid-shaped $\mathrm{P}_{\mathrm{aw}}$-t curve may be observed, leading to difficulties for analysis using visual inspection. Finally, the distribution of SI (linear, downward concavity, or upward concavity) may differ in other types of subjects, such as those with ARDS. ${ }^{17,18}$

\section{Conclusion}

This study demonstrates that SI can be accurately and reliably assessed by visually inspecting waveforms on the ventilator screen. This simplified method may help care providers use SI in clinical practice or in large trials for personalized mechanical ventilation.

\section{ACKNOWLEDGMENTS}

We thank Mr Dawei Wu for his excellent drawing of the ventilator screen in Figure 1.

\section{REFERENCES}

1. Slutsky AS, Ranieri VM. Ventilator-induced lung injury. N Engl J Med 2014;370(10):2126-2136.

2. Rocco PR, Dos Santos C, Pelosi P. Pathophysiology of ventilator-associated lung injury. Curr Opin Anaesthesiol 2012;25(2):123-130.

3. Brower RG, Matthay MA, Morris A, Schoenfeld D, Thompson BT, Wheeler A; on behalf of the acute Respiratory Distress Syndrome Network. Ventilation with lower tidal volumes as compared with traditional tidal volumes for acute lung injury and the acute respiratory distress syndrome. N Engl J Med 2000;342(18): 1301-1308.

4. Mercat A, Richard JC, Vielle B, Jaber S, Osman D, Diehl JL, et al.; on behalf of Expiratory Pressure (Express) Study Group. Positive end-expiratory pressure setting in adults with acute lung injury and acute respiratory distress syndrome: a randomized controlled trial. JAMA 2008;299(6):646-655.

5. Brochard L, Martin GS, Blanch L, Pelosi P, Belda FJ, Jubran A, et al. Clinical review: Respiratory monitoring in the ICU-a consensus of 16. Crit Care 2012;16(2):219.

6. Henderson WR, Chen L, Amato MBP, Brochard LJ. Fifty years of research in ARDS. Respiratory mechanics in acute respiratory distress syndrome. Am J Respir Crit Care Med 2017;196(7):822-833.

7. Ranieri VM, Zhang H, Mascia L, Aubin M, Lin CY, Mullen JB, et al. Pressure-time curve predicts minimally injurious ventilatory strategy in an isolated rat lung model. Anesthesiology 2000;93(5): 1320-1328.

8. De Perrot M, Imai Y, Volgyesi GA, Waddell TK, Liu M, Mullen JB, et al. Effect of ventilator-induced lung injury on the development of reperfusion injury in a rat lung transplant model. J Thorac Cardiovasc Surg 2002;124(6):1137-1144.

9. Gama de Abreu M, Heintz M, Heller A, Széchényi R, Albrecht DM, Koch T. One-lung ventilation with high tidal volumes and zero positive end-expiratory pressure is injurious in the isolated rabbit lung model. Anesth Analg 2003;96(1):220-228.

10. Grasso S, Terragni P, Mascia L, Fanelli V, Quintel M, Herrmann P, et al. Airway pressure-time curve profile (stress index) detects tidal recruitment/hyperinflation in experimental acute lung injury. Crit Care Med 2004;32(4):1018-1027.

11. Carvalho AR, Spieth PM, Pelosi P, Vidal Melo MF, Koch T, Jandre FC, et al. Ability of dynamic airway pressure curve profile and elastance for positive end-expiratory pressure titration. Intensive Care Med 2008;34(12):2291-2299.

12. Pan C, Tang R, Xie J, Xu J, Liu S, Yu T, et al. Stress index for positive end-expiratory pressure titration in prone position: a piglet study. Acta Anaesthesiol Scand 2015;59(9):1170-1178.

13. Grasso S, Stripoli T, De Michele M, Bruno F, Moschetta M, Angelelli G, et al. ARDSnet ventilatory protocol and alveolar hyperinflation: role of positive end-expiratory pressure. Am J Respir Crit Care Med 2007;176(8):761-767.

14. Huang Y, Yang Y, Chen Q, Liu S, Liu L, Pan C, et al. Pulmonary acute respiratory distress syndrome: positive end-expiratory pressure titration needs stress index. J Surg Res 2013;185(1):347-352.

15. Terragni PP, Filippini C, Slutsky AS, Birocco A, Tenaglia T, Grasso $\mathrm{S}$, et al. Accuracy of plateau pressure and stress index to identify 


\section{Ventilator WAVEForms AND STRESS INDEX}

injurious ventilation in patients with acute respiratory distress syndrome. Anesthesiology 2013;119(4):880-889.

16. Chiumello D, Cressoni M, Carlesso E, Caspani ML, Marino A, Gallazzi E, et al. Bedside selection of positive end-expiratory pressure in mild, moderate, and severe acute respiratory distress syndrome. Crit Care Med 2014;42(2):252-264.

17. Terragni PP, Rosboch GL, Lisi A, Viale AG, Ranieri VM. How respiratory system mechanics may help in minimising ventilatorinduced lung injury in ARDS patients. Eur Respir J Suppl 2003;42: $15 \mathrm{~s}-21 \mathrm{~s}$.
18. Terragni P, Bussone G, Mascia L. Dynamic airway pressure-time curve profile (stress index): a systematic review. Minerva Anestesiol 2016;82(1):58-68.

19. Viera AJ, Garrett JM. Understanding interobserver agreement: the kappa statistic. Fam Med 2005;37(5):360-363.

20. Harper R, Reeves B. Reporting of precision of estimates for diagnostic accuracy: a review. BMJ 1999;318(7194):1322-1323.

21. Formenti P, Graf J, Santos A, Gard KE, Faltesek K, Adams AB, et al. Non-pulmonary factors strongly influence the stress index. Intensive Care Med 2011;37(4):594-600.

This article is approved for Continuing Respiratory Care Education credit. For information and to obtain your CRCE

(free to AARC members) visit 